\title{
The effect of non-opioid analgesics on opioid use in general anesthesia
}

Alexander Avidan, MD, Matan J Cohen MD, Charles Weissman, MD, Phillip D. Levin, MDBChir

Department of Anesthesiology and Critical Care Medicine, Hadassah - Hebrew University Medical Center, Jerusalem Israel.

General Intensive Care Unit, Shaarei Zedek Medical Center

\section{Background}

Use of non-opioid analgesics (NOA) has been shown to reduce the requirement for perioperative opioids, and thus potentially decrease opioid side effects. This study examined longitudinal trends in NOA use and associated changes in opioid administration.

\section{Methods}

Data on opioid and NOA (dipyrone and ketorolac) use in adults during general anesthesia (without additional regional anesthesia) were retrieved from the computerized anesthesia information system for April 2007 to September 2015. Temporal changes in NOA and opioid use and dosages were examined.

\section{Results}

Data from 61,646 surgeries were analysed. NOA administration increased linearly from 333/1431 (23.3\%) patients (second quarter 2007) to $1381 / 1920$ (68.6\%) patients (third quarter 2015, $\mathrm{p}<0.001$ ). Mean morphine dose/patient was similar with or without NOA (with NOA: 12024 patients, 10.1 $\pm 1.3 \mathrm{mg}$; without NOA: 8961 patients, 9.6 $\pm 1.0 \mathrm{mg}, \mathrm{p}=0.66$ ). Mean morphine dose per patient declined over time at a similar rate for patients who did or did not receive NOA (Figure 1). Mean fentanyl dose per patient was lower in patients receiving NOA than in patients not receiving NOA (with NOA: 29,530 patients, $184 \pm 11 \mu$; without NOA: 25,422 patients, $247 \pm 21 \mu \mathrm{g}, \mathrm{P}<0.001)$. The mean fentanyl dose per patient decreased over time for patients receiving NOA, while it increased for those not receiving NOA (Figure).
Mean Doses of Opioids with or without Non-Opioid

Analgesics for General Anesthesia over 8.5 Years

350

35

300

30

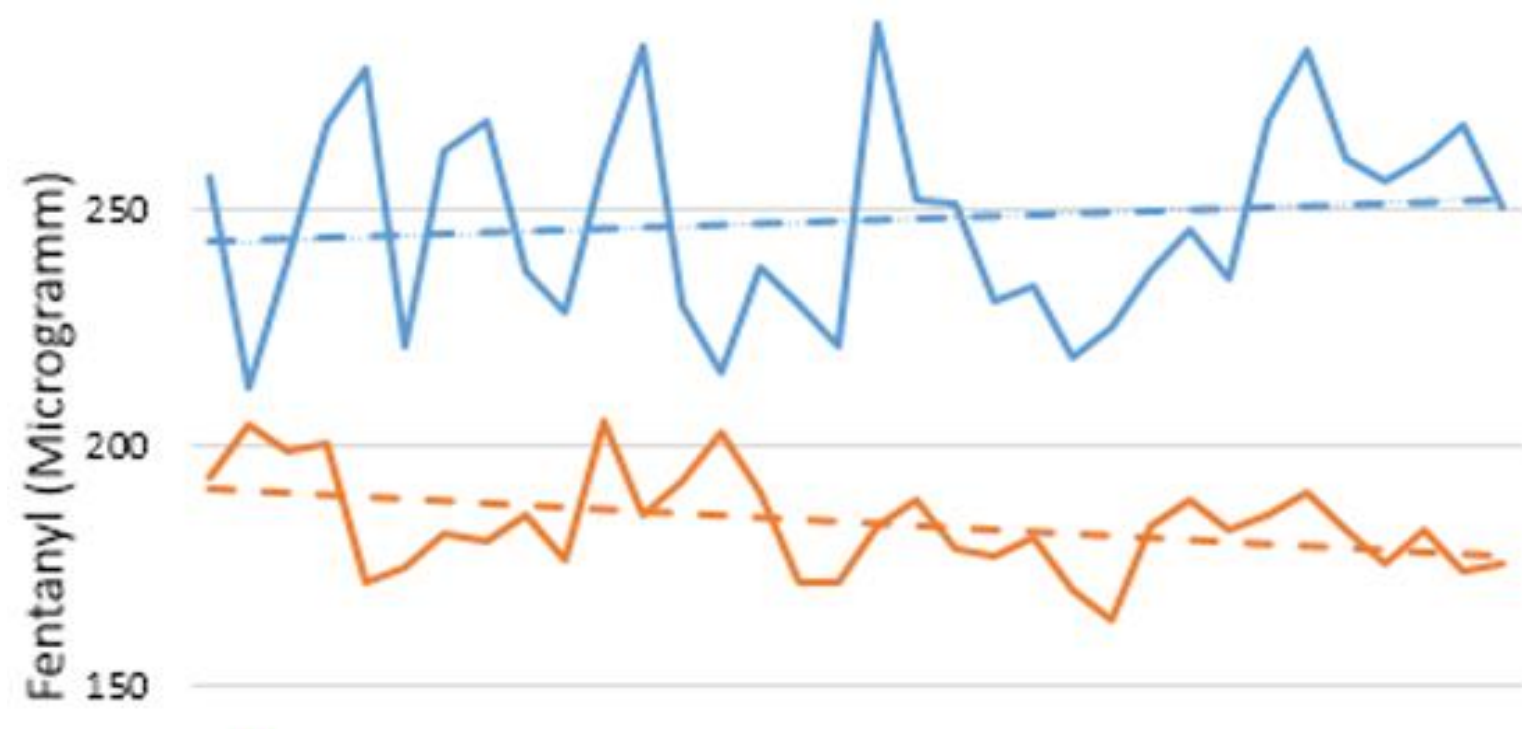

100

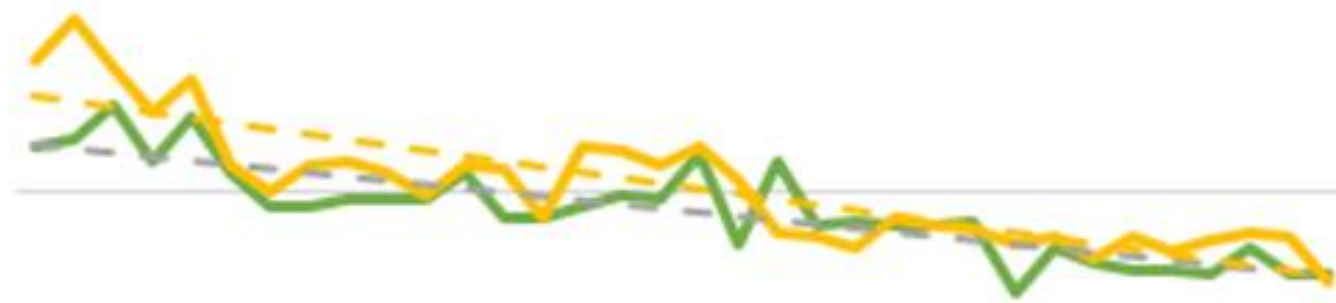

-Fentanyl without NOA

50 - Fentanyl with NOA

Morphine without NOA

Morphine with NOA

0

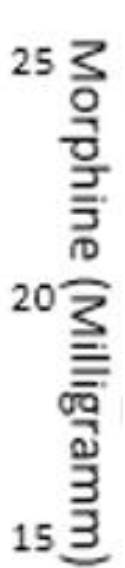

10

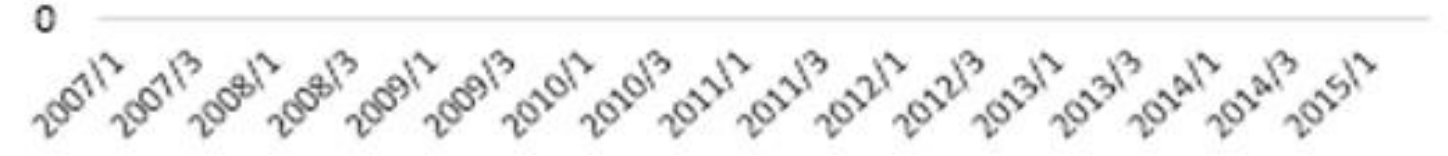

\section{Conclusions}

NOA administration in general anesthesia increased significantly over 8.5 years. NOA use was not associated with a change in morphine dosing. Fentanyl dose decreased over time in patients receiving NOA, but increased in patients not receiving NOA. This might reflect case selection - e.g. patients not planned for extubation may have received higher fentanyl doses and not be candidates for NOA. These findings suggest that factors other than NOA administration influenced opioid dosing. 